\title{
Wie viel Streiten gibt es in Streitgesprächen? Eine textlinguistische und sprachorientierte Untersuchung am Beispiel von Streitgesprächen in der Presse
}

\section{Einführung}

Den Gegenstand des vorliegenden Beitrags bilden sog. Streitgespräche, die sich in Medien wie Rundfunk, Fernsehen und Presse als eine Art Interview etabliert haben. Wie ihre Bezeichnung bereits anzeigt, soll es sich bei dieser Art von Gespräch um einen kontroversen Austausch von Meinungen zwischen Gesprächsteilnehmern handeln. Die Voraussetzung dafür sind ihre Meinungsunterschiede. In den meisten medial ausgetragenen Streitgesprächen werden vorwiegend Experten oder Politiker mit stark differierenden Auffassungen von einem Moderator um eine Stellungnahme zu bestimmten Sachverhalten gebeten. Wie zu vermuten ist, ergibt sich - wo zwei oder mehrere gegensätzliche Meinungen aufeinandertreffen - leicht ein Grund zum Streiten. Der Beitrag soll Streitgespräche kurz charakterisieren und Auskunft darüber geben, wie Streit innerhalb dieser Gespräche ausgetragen wird - als schroffer Schlagabtausch oder milder und höflicher Meinungsaustausch. In meinem Artikel möchte ich Streitgespräche als eine besondere Art des Interviews in der Presse darstellen und auf ihre Merkmale verweisen. Des Weiteren werden sprachliche Mittel und Phänomene dargelegt, die die Erfüllung einiger Funktionen belegen, die als typisch für Streitgespräche gelten können. Dabei soll vor allem auf folgende Fragen eingegangen werden: 
- Wie kann ein Gespräch und ein Streitgespräch aus textlinguistischer Perspektive charakterisiert werden?

- Was macht ein Streitgespräch aus? Was entscheidet, ob ein Streitgespräch oder ein herkömmliches Interview vorliegt?

- Welche sprachlichen Mittel und welches Sprachverhalten können als typisch für Streitgespräche gelten?

Zwar impliziert der Begriff „Gespräch“ primär seine mündliche Realisierung in Medien wie Rundfunk und Fernsehen, aber die von mir vorzunehmende Untersuchung bezieht sich auf ausgewählte Streitgespräche, die von der „Spiegel“Redaktion durchgeführt und im Magazin „Der Spiegel“ abgedruckt wurden. Es handelt sich somit um Streitgespräche, die am Beispiel des Mediums Presse charakterisiert werden.

\section{Streiten und Konflikt}

Meine Erwägungen in diesem Bereich möchte ich mit der Erläuterung des Begriffs „Streiten“ beginnen. Hierzu führe ich die Bedeutungserklärung des Lexems streiten des Duden-Universalwörterbuchs an:

1. mit jemandem Streit haben, in Streit geraten; sich mit jemandem in oft hitzigen Wortwechseln, oft auch in Handgreiflichkeiten heftig auseinandersetzen; sich zanken;

2. heftig über etwas diskutieren und dabei die unterschiedlichen, entgegengesetzten Meinungen gegeneinander durchsetzen wollen;

3. (gehoben) kämpfen;

4. (veraltet) eine kriegerische Auseinandersetzung führen (Kunkel-Razum et al. 2007:1631).

Die erste und die zweite Bedeutungserklärung scheinen in dem untersuchten Bereich besonders relevant zu sein, weil sie auf den verbalen Charakter des Streits hindeuten. Beim Streiten und bei einem Streitgespräch soll es sich dementsprechend um einen hitzigen Wortwechsel handeln, der von jeder der beteiligten Parteien mit dem Ziel vorgenommen wird, ihren Standpunkt unter verschiedenen und einander widersprechenden Meinungen durchzusetzen. Diese Definition erfasst recht genau das Ziel und die Hauptfunktion eines Streitgespräches.

Apeltauer (1977:36) definiert Streit ebenfalls als „,eine verbale Konfliktform, die sich durch hohe emotionale Beteiligung beider Partner auszeichnet und primär auf der Beziehungsebene abläuft". In der von Apeltauer angeführten Definition kommt die emotionale Beteiligung zum Tragen, die Streitgespräche von anderen Interviews, in denen nur eine Person befragt wird, unterscheidet. Diese emotionale 
Anteilnahme wird tatsächlich in der Analyse bestätigt, indem auf die große Klasse emotiver Ausdrücke hingewiesen wird. Die ebenfalls von Apeltauer erwähnte Beziehungsebene betrifft indessen das Image der an einem Gespräch Beteiligten.

Der mit Streiten zusammenhängende Begriff „Konflikt“ wird im Vergleich zu „Streiten“ weiter ausgelegt. In Anlehnung an Mack/Snyder (1957) definiert Gruber (1996:17) am Beispiel von Fernsehgesprächen eine Liste von Merkmalen einer Konfliktsituation. Vor dem Hintergrund eines Streitgespräches scheint in diesem Kontext Folgendes von Bedeutung zu sein:

- Konfliktsituationen erfolgen unter mindestens zwei Parteien.

- Das Ziel einer Konfliktsituation ist es, ,die andere(n) Partei(en) zu zerstören, zu verletzen, zu frustrieren oder anders zu kontrollieren. In einer Konfliktrelation kann eine Partei nur auf Kosten der anderen zu einem Erfolg kommen" (ebd.).

- Konflikt erfordert eine Interaktion, die eine Aktion und Reaktion verlangt.

- Eine Konfliktrelation setzt voraus, dass eine Partei Kontrolle und Position, sowie Macht erhält.

Die Untersuchung von Konfliktsituationen unter linguistischem Aspekt ist besonders in Bezug auf Streitgespräche von Bedeutung. Bezogen auf Richter (1979), Schwitalla (1987) und Bliesener (1984) stellt Gruber (1996:20-21) verschiedene Typen von Konfliktsituationen dar. Im Kontext der Streitgespräche ist besonders zwischen Meinungs- und sozialem Konflikt zu differenzieren. Der erste betrifft die Diskrepanz bezüglich der Meinungen der beteiligten Personen, der zweite bezieht sich indessen auf Unstimmigkeiten in der Bewertung und der sozialen Einordnung der gegebenen Sachverhalte (bezogen u.a. auf Wahrheit). Wenn man dazu noch den Aspekt des Images berücksichtigt, können auch Beziehungskonflikte ausgesondert werden, die das Image der an einem Gespräch teilnehmenden Person direkt betreffen, d.h. das Image bedrohen oder es schonen.

Die oben angeführten Erwägungen zu Streit, Konflikt und Image stimmen mit der Definition des Streitgespräches von Schwitalla (1987) überein. Als Streitgespräch wird hier ein Dialog definiert, in dem „im Zuge einer Auseinandersetzung über etwas nach den Erwartungen eines Angesprochenen zu wenig Rücksicht auf sein privates oder öffentliches Ansehen genommen wird, so dass er sich gegen die Verletzung seines Images wehrt" (Schwitalla 1987:108). Dem Streitgespräch liegt somit Imageverletzung und gleichzeitig der Versuch der Imageschonung zugrunde. Bezogen auf die Theorie von Goffman (1955) pointiert Schwitalla (1996:282), dass ,die Beteiligten bemüht sind, ein 'image of self' zu gewährleisten, dem gesellschaftlich positive Werte inhärent sind“. Darunter werden Handlungen und sprachliches Verhalten verstanden, durch die Imageverletzungen vermieden werden können. Mit diesen Handlungen ist auch der Begriff des positiven und negativen Gesichts verbunden. Das positive Gesicht zielt auf „Anerkennung und Bestätigung 
des Selbstbildes“ (Lüger 2014:42), das negative Gesicht bezieht sich indessen auf „den Wunsch eines Individuums, eine bestimmte Distanz, einen Freiraum, [...] zu bewahren“ (ebd.). Die Kommunikationsteilnehmer - besonders interviewte Personen - sind also bemüht, sich in einem guten Licht darzustellen. Sie möchten anerkannt werden, sich dabei aber einen bestimmten Freiraum bewahren.

Im Rahmen meiner Untersuchung möchte ich mich der Auffassung des Streitgespräches in Spiegel (2011:17) anschließen, die Streitgespräche folgendermaßen definiert: „,[Ein] Streitgespräch ist eine Form der verbalen Austragung von Konflikten, die keine manifesten Partnerdiskriminierung aufweist und die in der Regel in den Medien in Form von Disputen stattfindet". Zudem verweist Spiegel auf bestimmte Unterschiede in privaten und öffentlichen Streitgesprächen, in die u.a. Streitgespräche in der Presse fallen. Das Streiten ist eine Domäne des privaten Bereiches, in dem es sich häufiger abspielt. Gesellschaftlich offene Streitgespräche sind durch Standards und Verhaltenserwartungen determiniert, die von den Kommunikationsteilnehmern verfolgt werden müssen. Die sprachlichen Grenzen, Verhaltensmuster und Handlungsmöglichkeiten in privaten Streitgesprächen reichen somit viel weiter, weil diese von der Öffentlichkeit nicht wahrgenommen und bewertet werden (vgl. Spiegel 2011:20).

\section{Streitgespräch und Interview in der Presse}

Im nächsten Schritt möchte ich kurz auf Gesprächstypologien aus sprach- und medienwissenschaftlicher Perspektive eingehen. Sprachwissenschaftlich zählen Henne/Rehbock (2001:24) Mediengespräche und Interviews zu einer Subklasse, die nach dem Kriterium der Privatheit/Öffentlichkeit definiert wird. Mediengespräche und Interviews erfolgen nach diesem Kriterium öffentlich. Dabei entsteht die Frage, wie sich Mediengespräche von Interviews unterscheiden. Das „Mediengespräch“ betrachte ich als allgemeinen Begriff, der alle Gespräche in den Medien Presse, Rundfunk und Fernsehen umfasst. $\mathrm{Zu}$ ihnen werden z.B. Talkshows, Rundfunk-Interviews, Höreranrufe und daraus entstehende Gespräche gezählt. Solche Gespräche unterscheiden sich von natürlichen Alltagsgesprächen im Geschäft, unter Freunden, in der Familie oder in der Arbeit, denn „Gespräche in den Massenmedien stellen einen Gesprächstypus sui generis dar" (Burger 2001:1492). Der größte Unterschied zwischen den genannten Typen besteht darin, dass Mediengespräche einem breiten Publikum vermittelt werden, im Gegensatz zu Alltagsgesprächen, die privaten Charakter haben und nicht öffentlich erfolgen.

Einen Sonderfall der Mediengespräche bilden Gespräche in der Presse, in der sie nicht mehr mündlich, sondern verschriftlicht erscheinen. Zu solchen Gesprächen stellt Burger (2001:1494) fest: „In der Presse werden Gespräche häufig nicht tale quale, d.h. als Textsorte 'Pressegespräch' vermittelt, sondern in stark bearbeiteten Mischformen [...], bei denen zwar der Gesprächscharakter der Originalsituation 
noch durchscheint, doch die einzelnen Gesprächsbeiträge des Interviewten fragmentiert und redaktionelle Texte neu zu einem monologisch-dialogischen Textganzen kombiniert werden". Eine eigene Sorte von in der Presse veröffentlichten Gesprächen stellen Presseinterviews dar. Sie sind Verschriftlichungen zunächst mündlicher Texte, die meist auf der akustischen Aufzeichnung einer medial mündlichen Gesprächssituation basieren. Das aufgenommene Gespräch wird transformiert und pressespezifisch unter Einhaltung sprechsprachlicher Phänomene bearbeitet (vgl. Burger 2001:1496), wohl mit dem Ziel, den primären mündlichen Charakter des Gespräches zu bewahren. Einen bedeutenden Beitrag zur Beschreibung des medialen Interviews aus der sprachwissenschaftlichen Perspektive leistet Schwitalla (1979), der Medieninterviews nach dem Kriterium der zu interviewenden Person klassifiziert, und zwar in Politiker-, Experten- und Starinterviews. In Anlehnung an Schwitalla (1979:164-165) kann das Interview als dialogische Darstellungsform klassifiziert werden, deren Struktur durch Frage-Antwort-Sequenzen gekennzeichnet ist. Das Interview als Textsorte betrifft Fragen des öffentlichen Interesses (beispielsweise im Falle der Interviews mit Experten oder Politikern) oder den privaten Bereich (bei Prominenten). In Interviews dominiert eine argumentative Themenentfaltung, die auch in Streitgesprächen deutlich zum Tragen kommt.

Aus medienwissenschaftlicher Perspektive indessen ist ein Presseinterview die journalistische Darstellungsform schlechthin. Das durchgeführte Gespräch erscheint als ein im Nachhinein ,geformtes“ bzw. ,gestaltetes“ Interview (Haller 2013:123). Die Klassifikation der Presseinterviews kann aus dieser Perspektive nach dem Kriterium des abgehandelten Themas vorgenommen werden. Danach lassen sich Interviews zur Sache (Aussagen zu bestimmten Fakten), Interviews zur Meinung (Bewerten eines Problems) und Interviews zur Person (Porträt) unterscheiden (vgl. Haller 2013:25). Bei Streitgesprächen handelt es sich somit vorwiegend um Interviews zur Meinung, oder präziser gesagt, um Interviews zu erheblich differierenden Meinungen.

Die zu untersuchenden Streitgespräche sind also eine besondere Form des Presseinterviews. Charakteristisch für diese Gespräche ist die Befragung zweier Interviewter zu einem heiklen oder brisanten Thema, für das ein gewisses Publikumsinteresse besteht. Die Meinungen der Befragten müssen eindeutig voneinander abweichen, damit tatsächlich ein Gespräch mit Streitcharakter zustande kommt. Wie schon vorausgeschickt, hängen mit dem Streitgespräch als Interviewform auch die Begriffe Image und Selbstdarstellung zusammen. Ein Streitgespräch in der Presse setzt voraus, dass konträre Meinungen geäußert werden, ohne darauf einzugehen, wer Recht hat und wer in dem Streit überlegen ist. Die Interviewten möchten aber auf jeden Fall das Publikum, also die Leser, von ihren Argumenten überzeugen und diese als die (einzig) richtigen wahrgenommen sehen. Dieses Überzeugen erfolgt nicht immer glatt; oft wird versucht, die andere Partei bloßzustellen und in einem schlechten Licht erscheinen zu lassen, um das eigene Image zu wahren. 
Auf den Streitcharakter der analysierten Interviews wird nicht selten in der von der Redaktion verfassten Einführung zum Gespräch hingewiesen. So ist die Rede von einem Schlagabtausch oder von großen Gegnern, die zum Gespräch eingeladen wurden; von Politikern, deren Vorstellungen aufeinanderprallen. Den Charakter solcher Gespräche offenbaren darüber hinaus auch explizit Verben wie widersprechen oder streiten:

(1) Machen digitale Spiele süchtig? Wie viel Zeit am Computer ist gesund? Ein Schlagabtausch zwischen dem Suchttherapeuten Klaus Wölfling und dem Spielelobbyisten Martin Lorber über die Grenze zwischen Freiheit und Krankheit (Interview Nr. 4).

(2) Der Gesundheitsökonom Karl Lauterbach und der Ärztekammerpräsident Frank Ulrich Montgomery sind zwar beide SPD-Mitglieder, aber zugleich große Gegner, wenn es um die Bürgerversicherung geht (Interview Nr. 1).

(3) Der Chef der konservativen AfD Bernd Lucke, 50, und Linkspartei-Vizin Sahra Wagenknecht, 43, erklären die Euro-Rettung für gescheitert. Wenn es aber um die Lösung der Krise geht, prallen ihre Vorstellungen aufeinander (Interview Nr. 2).

(4) Der Ökonom Thomas Mayer wirft der EZB vor, die Zinsen niedrig zu halten - zulasten der Sparer. Sein Kollege Peter Bofinger widerspricht (Interview Nr. 7).

(5) Was ist die Geburt, total normal oder ein Risiko? Was gibt Sicherheit, die gut ausgestattete Klinik oder die entspannte Atmosphäre? Und sollte die Politik sich einmischen? Darüber streiten die Hebamme Martina Klenk und der Gynäkologe Volker Ragosch (Interview Nr. 12).

Dieses Verfahren ist allerdings keine bindende Regel - in den zwölf analysierten Gesprächen fanden sich solche Merkmale in fünf Texten.

Besonders wichtig in dieser Form von Gespräch ist die Rolle des Interviewers. Die Lektüre der Streitgespräche zeigt eindeutig, dass die Atmosphäre und der Charakter eines Streits erzielt werden, wenn die fragende Person in den Austausch von Argumenten nur soweit erforderlich eingreift und den Verlauf des Gespräches den Interviewten überlässt. Dem Interviewenden kommt eher die Rolle des Moderators zu, indem er das Gespräch dann in die richtigen Bahnen zurücklenkt, wenn es tatsächlich notwendig ist. Erfolgt das Gespräch aber nach dem Muster:

Frage

Antwort von Person A

Antwort von Person B

verliert das Gespräch weitgehend seinen Streitcharakter. 


\section{Analyse}

In meinem Beitrag wurden zwölf Streitgespräche einer Analyse unterzogen. Die in ihnen abgehandelten und für Differenzen sorgenden Themen betreffen aktuelle politische und soziale Probleme. Ein Verzeichnis der Streitgespräche und ihrer Teilnehmer ist unten angefügt. Aus den untersuchten Gesprächen wird ersichtlich, dass in ihnen Elemente vorhanden sind, die den Streit anheizen oder entschärfen können. Spiegel (2011) spricht in diesem Zusammenhang von Eskalation und Deeskalation im Verlauf eines Gespräches. Diese Elemente verleihen dem Streitgespräch „die streittypische Form und prägen [...] die Modalität Streit“" (Spiegel 2011:11). Da die Grundlage der Untersuchung verschriftlichte Texte bilden, kann die Eskalation bzw. Deeskalation eines Gespräches hier lediglich auf lexikalischer Ebene erfasst werden. Nicht-verbales Verhalten, Stimme, Intonation können leider nicht berücksichtigt werden. Die Eskalation wird in dieser Hinsicht vor allem durch den "Gebrauch expressiver, meist negativ bewertungshaltiger Lexeme“ (Spiegel 2011:25) signalisiert. Mittel der Deeskalation im Gespräch sind indessen u.a. eine ,entsprechende Wahl des Ausdrucks, Eingeständnisse, Entschuldigungen, Selbstvorwürfe, Honorierung des Interaktionspartners" (ebd.).

Im Folgenden möchte ich der Frage nachgehen, wie sich der Streitcharakter auf sprachlicher Ebene widerspiegelt. Wie schon gesagt, unterscheiden sich Streitgespräche von anderen Presseinterviews durch heftigen Meinungsaustausch und die Anzahl der Interviewten. Vor dem Hintergrund der sprachlichen Realisierung von Streitgesprächen sind folgende Fragen zu beantworten:

- Woran erkennt man den Streitcharakter eines Gespräches bzw. was indiziert den Streitcharakter?

- Durch welche sprachlichen Strategien und in welchen Gesprächsmomenten kommt das Streiten besonders zum Tragen?

- Woran erkennt man Bestreiten, Beleidigungen, Empörung oder Scheinhöflichkeit?

Dazu verweise ich auf bestimmte in Streitgesprächen wiederkehrende Konstruktionen und Phänomene und ziehe zur Veranschaulichung kurze Belege mit Quellenverweis heran. Die uns hier interessierenden Stellen sind zusätzlich fett gesetzt.

In der vorgenommenen Recherche konnten folgende Sprachverhaltensweisen und Indikatoren des Streitens ermittelt werden:

- Explizites Bestreiten

Das wichtigste Merkmal und zugleich Ziel eines Streitgespräches ist ein heftiger Wortwechsel, eine harte Diskussion und der Austausch von Argumenten. Dieses Ziel erreicht man durch eine entsprechende Darstellung eigener Einstellungen, 
aber auch dadurch, dass man Argumente des Opponenten zurückweist und sie bestreitet. Unten werden einige Belege präsentiert, in denen das Bestreiten entweder explizit oder implizit erfolgt. Das explizite Bestreiten in (1)-(6) erfolgt vor allem durch Verben aus dem semantischen Feld des Verbs bestreiten oder Adjektive in Verbindung mit dem Kopulaverb sein. Unter implizitem Bestreiten in (7)-(10) verstehe ich hingegeben Ausdrücke und Äußerungen, mit denen ein Sachverhalt nicht explizit für unzutreffend erklärt wird:

(1) Montgomery: Ich bestreite, dass deine Horrorzahlen stimmen (Interview Nr. 1).

(2) Lucke: Das bestreite ich. Aber die Höhe der Löhne ist nicht entscheidend. Wichtig ist, ob sie der Produktivität entsprechen (Interview Nr. 2).

(3) Bofinger: Das stimmt so nicht. Natürlich sind heute die langfristigen Zinsen im Vergleich zur Geldentwertung extrem niedrig. Das ist ein Riesenproblem für die private Altersvorsorge. Aber wer sein Geld auf dem Sparbuch anlegt, musste es schon in der Vergangenheit häufig erleben [...] (Interview Nr. 7).

(4) Klenk: Nein, man kann das Risiko nie vollends ausschalten. Auch Technik bietet keine absolute Sicherheit. Herr Ragosch redet wie ein typischer Schulmediziner, der den Frauen suggeriert, wenn man nur alle technischen Möglichkeiten nutze, sei man auf der sicheren Seite. Das stimmt aber nicht (Interview Nr. 12).

(5) Herrmann: Ich weise Sie lediglich darauf hin, dass ich nicht Politiker in Griechenland bin und Ihre Behauptungen nicht überprüfen kann. Völlig unstrittig ist, dass im Mittelmeer niemand ertrinken darf. Aber genauso wenig kann die EU jedem schiffbrüchigen Migranten Asyl gewähren (Interview Nr. 11).

(6) Nogge: Ich hielte es aber auch für falsch, wenn der Staat den Zoos vorschreiben würde, welche Tiere sie halten dürfen und welche nicht (Interview Nr. 6).

(7) Fratzscher: Das sehe ich ganz anders. Die EZB muss die Wirtschaft mit Liquidität versorgen, damit die Verbraucher einkaufen und die Unternehmen investieren können (Interview Nr. 3).

(8) Herrmann: Das ist jetzt völlig daneben. Wir haben auf dem Parteitag intensiv über das Thema Asyl diskutiert. Und wir waren uns einig, dass wir Fluchtursachen letztlich vor Ort bekämpfen müssen. Durch Entwicklungshilfe in Afrika und im Nahen Osten (Interview Nr. 11).

(9) Juncker: Nun mal langsam. Wir haben in der EU unter meinem Vorsitz 1997 beschlossen, die Zinssteuern in Europa zu harmonisieren. Wir haben einen Kodex gegen unfairen Steuerwettbewerb aufgestellt. [...] Ich werde mir das nicht bieten lassen (Interview Nr. 5). 
(10) Mayer: Ich wäre strikt dagegen, die Haushaltsdisziplin aufzugeben. Wir sehen doch immer wieder, wie der Staat das Geld für Konsum und soziale Wohltaten ausgibt. Und dort, wo er investiert, geht es häufig schief, Stichwort Berliner Flughafen BER (Interview Nr. 7).

In den untersuchten Streitgesprächen konnten auch Stellen ermittelt werden, in denen die Meinungen der Interviewten übereinstimmten, was als Signal der Deeskalation dienen kann. Oft impliziert eine solche Zustimmung aber dennoch eine Absicherung und Distanzierung, die vor allem durch den Ausdruck na ja deutlich wird:

(11) Wölfling: Na ja, da gibt es doch genug Möglichkeiten, das zu umgehen (Interview Nr. 4).

(12) Spahn: Na ja, die Jüngeren sind im Präsidium wirklich nicht überrepräsentiert. Das ist ein Grund, warum ich antrete, unterstützt von der Jungen Union und der Mittelstandsvereinigung in der CDU (Interview Nr. 9).

- ja-aber-Konstruktion

Ein sehr häufiges sprachliches und rhetorisches Mittel in den untersuchten Texten der Streitgespräche sind ja-aber-Konstruktionen. Sie werden nach folgendem Muster konstruiert:

vorläufiges, anscheinendes Zustimmen (ja), Gegenargument (aber).

So signalisiert eine Gesprächspartei vorläufige Zustimmung und gibt vor, mit dem anderen Gesprächsteilnehmer einverstanden zu sein. Gleich darauf folgen Gegenargumente, die die eigentliche These des Gegners in Frage stellen sollen. In jedem der untersuchten Interviewtexte wurde eine ja-aber-Konstruktion eingesetzt:

(13) Wölfling: Dass Computerspiele positive Seiten haben, eine kreative Kraft, das will ich gar nicht bestreiten. Aber das ändert für mich nichts daran, dass sie auch ein Suchtmittel sind - wohl näher dran am Glücksspiel als an Alkohol und Nikotin, aber nicht weniger gefährlich (Interview Nr. 4).

(14) Lorber: Sicherlich kann man über Computerspiele auch aus medizinischer Sicht sprechen, und die Erkenntnisse, die es da gibt, will ich gar nicht in Frage stellen. Im Gegensatz zu Herrn Wölfling bin ich kein Therapeut. Aber das wäre ungefähr so, als würde man eine WagnerOper vor allem unter dem Aspekt betrachten, ob der Schalldruck im Orchestergraben die Musiker schädigen könnte (Interview Nr. 4). 
(15) Bierdel: In absoluten Zahlen mag das stimmen. Aber gemessen an Einwohnerzahl und Wirtschaftskraft nimmt sich unser Einsatz für Menschen in Not bescheiden aus. Bis heute fällt der Bundesregierung als Antwort auf das Flüchtlingsdrama nicht sehr viel mehr ein, als Asylbewerber in Lager zu stecken ... (Interview Nr. 11).

(16) Nogge: Natürlich sollen Tiere im Zoo nicht leiden. Aber es macht doch einen Riesenunterschied, ob Sie einen Tierfilm sehen oder hautnah eine zwölfköpfige Gorillagruppe erleben, die auf der Wiese nach Nahrung sucht. Die persönliche Begegnung erlaubt ein unvergleichbares Erlebnis (Interview Nr. 6).

(17) Bofinger: Nach der Theorie haben Sie recht. Ich bringe die Formel auch meinen Studenten bei; sie steht sogar in meinem Lehrbuch. Aber die wirtschaftliche Realität ist eine andere. Höhere langfristige Zinsen gibt der Markt derzeit einfach nicht her (Interview Nr. 7).

(18) Fratzscher: Es stimmt, dass Geldanleger heute nur eine geringe Rendite bekommen. Aber es gibt Alternativen (Interview Nr. 3).

Solche Strukturen erfüllen m.E. zweierlei Funktionen: Zwar handelt es sich in dem untersuchten Bereich um Streitgespräche, aber die Kommunikationsteilnehmer möchten nicht als schroff oder unhöflich gelten. Sie sind sich bewusst, dass ihre Aussagen einem breiten Publikum vermittelt werden. Durch solche Konstruktionen können sie sich konziliant zeigen und zugleich eine höfliche Gesprächsatmosphäre (zumindest) anstreben. Sie dienen somit als Deeskalationsmarker. Auf der anderen Seite wirken so dargestellte Eigenargumente nachdrücklicher und überzeugender, weil mit ihnen Argumente des Gegenübers hinterfragt werden.

- Persönliche verbale Angriffe

Ein Streitgespräch macht ein heftiger Austausch von Meinungen und Argumenten aus. Ihr charakteristisches Merkmal ist auch der Umstand, dass sie nicht frei von verbalen persönlichen Angriffen sind, durch die Streitgespräche eskalieren können. Sie kennzeichnen sich zudem durch persönliche Anspielungen oder gar Beleidigungen. Dadurch wird versucht, die Hypothesen und Argumente des Partners zu widerlegen und somit seine Position zu schwächen. Da die untersuchten Streitgespräche öffentlich vermittelt werden, müssen solche Angriffe relativ mild erfolgen. Diese Funktion wird z.B. im Herunterspielen des Gegenübers oder in despektierlichen Äußerungen zur ausgeübten Funktion bzw. zum Beruf des Gegenübers deutlich:

(19) Lucke: Sie überschätzen sich. Meine Euro-Kritik stützt sich allein auf ökonomische Erkenntnisse. Was die Linke dazu sagt, habe ich nie verfolgt (Interview Nr. 2). 
(20) Klenk: Doch. Ich ertaste mit den Händen die Größe des Kindes. Ragosch: Geburtshilfe durch Handauflegen, das glauben Sie doch selbst nicht (Interview Nr. 12).

(21) Bode: Sie sind doch Jurist, Herr Wissmann. Dann sollten Sie eigentlich wissen, dass TTIP ein völkerrechtlicher Vertrag ist, der automatisch EU-Sekundärrecht aushebelt. Gesetze müssen dann TTIP-kompatibel sein (Interview Nr. 8).

(22) Grün: Sie sind aber gar kein Personenbeförderer, auch kein neuer. Sie sind ein Tourenmakler. Würden für Sie die Regeln des Taxigewerbes gelten, müssten Ihre Fahrer Sozialabgaben abführen, Lohnsteuer zahlen. Aber davor drücken Sie sich ja. Sie fordern ein neues Beförderungsgesetz, von den Pflichten wollen Sie nichts wissen (Interview Nr. 10).

- Konfrontation

In diesem Punkt werden Konfrontationen in Gestalt von Gegenüberstellungen abgehandelt, die ebenfalls ein Element des Streitens darstellen. Diese erkennt man am deutlichsten an den Gegensätzen ,ich-du“ oder ,ich-andere“, eventuell auch am konfrontativen Vergleich mit anderen Personen:

(23) Schulz: Ich würde politische Lösungen nicht mehr nur im traditionellen Institutionsgefüge der EU suchen. Ich würde die Kommission soweit es geht öffnen. Brüssel darf sich nicht in jede Kleinigkeit einmischen. Was immer auf kommunaler, regionaler oder nationaler Ebene geregelt werden kann, sollte dort geschehen. Ich bin ein Mann des Parlaments, ein Mann der Bürgerinnen und Bürger. Juncker ist ein Vertreter der Exekutive (Interview Nr. 5).

(24) Mayer: Für Sie mag das stimmen, Herr Bofinger, aber als Normalverdiener geht man mit einer Immobilie ein sehr großes Risiko ein (Interview Nr. 7).

(25) Juncker: Unsinn. Ich bin doch keiner, der sich nur in der Exekutive auskennt. Ich habe mich immer intensiv mit dem Europäischen Parlament ausgetauscht und in vielen Fragen gemeinsame Lösungen gesucht. Aber es ist auch kein Nachteil, die Befindlichkeiten und Interessen der Nationalstaaten im Europäischen Rat zu kennen. Das tue ich besser als Martin Schulz (Interview Nr. 5).

Ein solches Verfahren ist durch Kontraste und Konfrontation besonders anschaulich und wirkungsvoll. In ihm wird die Position der anderen Partei geschwächt und deren Image beschädigt. 
- Anreden als Element des Streitens

Eine Anrede als solche wird als Höflichkeitsindikator und Element der sozialen Zuordnung verwendet. Eine interessante, obgleich nicht immer eindeutige Rolle spielen Anreden in Streitgesprächen. Hier werden sie oft ironisch und scheinhöflich zur Betonung des eigenen Standpunktes verwendet. Sie dienen der Bekräftigung eigener Argumente und unterstreichen den Streitcharakter einer Aussage. Eine Anrede kann außerdem ironisch verwendet werden, wie etwa in dem ironisch eingesetzten Titular-Adressativ Herr Professor:

(26) Bierdel: An den Rändern Europas wird jeden Tag massiv gegen die Menschenrechte und gegen das Völkerrecht verstoßen. Und Sie, Herr Herrmann, sagen: Das ist mir egal. Hauptsache, in Deutschland kommen keine Flüchtlinge an (Interview Nr. 11).

(27) Schulz: Die Griechenland-Rettung war aber nicht besonders sozial. Und die haben Sie als Chef der Euro-Gruppe maßgeblich mitbestimmt, Herr Juncker. Wenn Sie durch Südeuropa touren, werden Sie merken, dass die Leute die EU in dieser Frage für extrem ungerecht halten (Interview Nr. 5).

(28) Mayer: Es stimmt, die Zentralbanken in den USA und in England gehen sogar noch weiter als die EZB, indem sie weiterhin selbst Anleihen aufkaufen, um den Zins zusätzlich nach unten zu drücken. Wir leben in einer Welt, in der sozusagen der Urmeter unseres Finanzsystems ständig manipuliert wird. So viel, Herr Bofinger, zum Thema Markt (Interview Nr. 7).

(29) Montgomery: Ich weiß es noch nicht. Aber falls ich SPD wählen sollte, lieber Karl, dann sicher nicht wegen dir und deiner Bürgerversicherung. In der Gesundheitspolitik bist du auf dem falschen Weg (Interview Nr. 1).

(30) Montgomery: Herr Professor, das ist jetzt aber sehr platt: Ich dachte, du fühltest dich einer gewissen wissenschaftlichen Objektivität verpflichtet (Interview Nr. 1).

- emotive Ausdrücke

Es wurde referiert, dass die emotionale Beteiligung eins der wichtigsten Merkmale von Streitgesprächen ist. Das Gespräch verläuft nicht immer so, wie von den Gesprächsteilnehmern erwünscht, was Emotionen großen Spielraum eröffnet. Dies wird besonders im Falle des Widerspruchs deutlich. In der vorgenommenen Untersuchung konnten sehr viele kurze und emotive Ausdrücke ermittelt werden, die vorwiegend dem Ausdruck eindeutigen Widerspruchs oder sogar der Empörung dienen. In Anlehnung an Lenk (2014:85ff.) konnten u.a. folgende Klassen emotiver Formulierungen ausgesondert werden, die den emotionalen Charakter von Streitgesprächen belegen: 
- Emotive Ausdrücke des Widerspruchs

(31) Herrmann: Unsinn! Die Situation in Syrien ist ohne Zweifel eine Katastrophe. Aber Deutschland hat in den vergangenen drei Jahren 70000 Syrer aufgenommen (Interview Nr. 11).

(32) Herrmann: Unsinn. Aber wir erleben nun mal einen gewaltigen Zustrom von Menschen, die null Chancen haben, hier bleiben zu können (Interview Nr. 11).

(33) Montgomery: Falsch. Deine sogenannte Bürgerversicherung würde das Problem der Zweiklassenmedizin in Wahrheit noch schlimmer machen (Interview Nr. 1).

(34) Lucke: Falsch. In Deutschland entsprechen die Löhne ungefähr der Produktivität der Arbeitnehmer (Interview Nr. 2).

(35) Lauterbach: Moment! Ich bin auch Mitglied der Ärztekammer. Und ich treffe eine ganze Reihe von Kollegen, die eine Bürgerversicherung gut fänden, weil es dann endlich keine Unterscheidung zwischen Patienten erster und zweiter Klasse mehr gäbe (Interview Nr. 1).

(36) Spahn: Moment mal. Die Schwarze Null, der ausgeglichene Bundeshaushalt, ist ein toller Erfolg. Dafür kämpfe ich, seit ich vor 19 Jahren in die Junge Union eingetreten bin. Keine neuen Schulden mehr zu machen ist gut für alle künftigen Generationen (Interview Nr. 9).

- Erstaunens- und Empörungssignal

(37) Bierdel: Großer Gott! Das sind doch olle Kamellen. Dieser Vorschlag wird seit Jahren hin und her gewälzt. Auch da geht es wieder nur darum, Flüchtlinge von Europa fernzuhalten. Die CSU spricht ja nicht umsonst von ,Flüchtlingsprävention“ (Interview Nr. 11).

- Zurückweisungssignale

(38) Fratzscher: Wie bitte? Wie kommen Sie auf diese Zahl? (Interview Nr. 3). (39) Bode: Wie kommen Sie denn darauf? Selbst nach einer Studie im Auftrag der Europäischen Kommission könnte das Handelsabkommen nur eine einmalige Niveauanhebung des EU Bruttoinlandsprodukts um 0,5 Prozent bringen - und das erst zehn Jahre, nachdem TTIP eingeführt wurde (Interview Nr. 8).

- Verärgerungsausdrücke

(40) Wagenknecht: ... wenn man entsprechend viel importiert. Wir Deutschen dagegen setzen das Geld, das wir mit unseren Exportüberschüssen 
verdienen, in den Sand - indem wir es in US-Hypothekenpapiere oder griechische Staatsanleihen investieren. Das ist doch Irrsinn (Interview Nr. 2).

(41) Wagenknecht: Wahlkampfhilfe? Das ist ja wohl ein Scherz. Nein, er hat sich nicht bedankt (Interview Nr. 2).

(42) Wissmann: Das ist Unfug. Eine gesetzgeberische Weiterentwicklung bleibt immer möglich. Es hängt doch alles davon ab, wie die Europäer die Verhandlungen führen (Interview Nr. 8).

(43) Montgomery: Das ist Blödsinn. Auf diesem Niveau sollten wir nicht diskutieren. Ich selbst bin angestellter Arzt im Krankenhaus; ich würde von einer Veränderung beim Honorar überhaupt nichts merken (Interview Nr. 1).

(44) Wagenknecht: Das ist doch Quatsch. In der deutschen Industrie wird weniger bezahlt als in der französischen (Interview Nr. 2).

\section{Zusammenfassung}

Streitgespräche sind eine besondere Form des Gespräches und zugleich eine besondere Form des Interviews. Laut der Definition des Duden-Universalwörterbuchs ist ein Streitgespräch ein „längeres, kontrovers geführtes Gespräch“ (Kunkel-Razum et al. 2007:1632). In ihm treffen zwei Interviewte mit verschiedenen Meinungen aufeinander. An den Begriff „Streitgespräch“ sind darüber hinaus Synonyme wie Schlagabtausch, verbale Auseinandersetzung und Meinungsverschiedenheit gebunden. Die Meinungsverschiedenheit spiegelt sich im Verlauf des Streitgespräches u.a. auf sprachlicher Ebene wider. Der Streitcharakter solcher Gespräche kommt durch verschiedene sprachliche und rhetorische Mittel zum Tragen, die vor allem dem Bestreiten und dem Ausdruck des Widerspruchs dienen. Im vorliegenden Beitrag habe ich versucht, auf Indikatoren des Streitens hinzuweisen. Die meisten dargestellten Phänomene dienen der Eskalation von Streitgesprächen in der Presse. In diesem Zusammenhang sei noch einmal auf Bestreiten, ironisch und beleidigend verwendete Anreden, verbale Angriffe und besonders auf emotive Ausdrücke verwiesen. Letztere signalisieren Verärgerung, Empörung oder eindeutigen Widerspruch und zeigen die emotionale Beteiligung der Interviewten.

Dargestellt wurden vorwiegend sprachliche Mittel, die dazu dienen, dem Gesprächspartner zu widersprechen und seine Argumente zu hinterfragen oder herunterzuspielen. Als charakteristisch für Streitgespräche haben sich dabei jaaber-Konstruktionen, krasse Gegenüberstellungen und sogar persönliche Verbalangriffe herausgestellt. Diese kommen in anderen Typen von Interviews, in denen nur eine Person interviewt wird, kaum vor. Abgesehen von der sprachlichen Seite und dem Sprachverhalten der Beteiligten wurde auf die Rolle des Interviewers 
hingewiesen. Je weniger er / sie in den Verlauf des Gespräches eingreift und seinen Verlauf den Interviewten überlässt, desto häufiger treten den Streitcharakter ausmachende Elemente auf.

Aus sprachwissenschaftlicher Perspektive sind Streitgespräche ein interessanter Gegenstand, denn über die dargestellten sprachlichen Indikatoren des Streitens hinaus eröffnen sie einen großen Spielraum für Image-Arbeit, Selbstdarstellung oder sogar Inszenierung der Interviewten. Zudem sind für Streitgespräche Überraschung, Ironie, verbaler Angriff und eine bestimmte Unerwartetheit bzw. Ungewissheit kennzeichnend, durch die diese Form von Gesprächen für die Leser besonders spannend zu sein scheint.

\section{Literatur}

APELTAUER Ernst, 1977, Drohen, in: Sprengel K./Bald W.D./Viethen H. (Hrsg.), Semantik und Pragmatik. Akten des 11. Linguistischen Kolloquiums Aachen 1976, Tübingen, S. 187-198.

BLIESENER Thomas, 1984, Gesprächskrisen. Entstehung und Bewältigung von Komplikationen in der Gesprächsführung, Opladen.

BURGER Harald, 2001, Gespräche in den Massenmedien, in: Brinker K./Antos G./Heinemann W./ Sager S.F. (Hrsg.), Text- und Gesprächslinguistik (2. Halbband), Berlin/New York, S. $1492-1505$.

GOFFMAN Erving, 1955, On face-work, in: Psychiatry 18, 1955, S. 213-231.

GOFFMAN Erving, 1975, Interaktionsrituale. Über Verhalten in direkter Kommunikation, Frankfurt am Main.

GRUBER Helmut, 1996, Streitgespräche. Zur Pragmatik einer Diskursform, Wiesbaden.

HALLER Michael, 2013, Das Interview. 5., völlig überarbeitete Auflage, München.

HENNE Helmut / REHBOCK Helmut, 2001, Einführung in die Gesprächsanalyse, Berlin/New York.

KUNKEL-RAZUM Kathrin et al. (Hrsg.), 2007, Duden - Deutsches Universalwörterbuch. 6., überarbeitete und erweiterte Auflage, Mannheim u.a.

LENK Hartmut E.H., 2014, Kommunikative Routineformeln in Zeitungskommentaren, in: Kolehmainen L./Lenk H.E.H./Tiittula L. (Hrsg.), Kommunikative Routinen - Formen, Formeln, Forschungsbereiche: Festschrift zum 65. Geburtstag von Prof. Dr. Irma Hyvärinen, Frankfurt am Main, S. 77-98.

LÜGER Heinz-Helmut, 2014, Meledicere et delectare? Unhöflichkeit als Persuasions- und Unterhaltungsstrategie, in: Lenk H.E.H./Suomela-Härmä E. (Hrsg.), Höflichkeit in Textsorten, Landau, S. $41-62$.

MACK Raymond W. / SNYDER Richard C., 1957, The Analysis of Social Conflict - Toward an Overview and Synthesis, in: Journal of Conflict Resolution 1, New York, S. 212-248.

RichTER Helmut, 1979, Konflikte in Dialogen, in: Heindrichs W./Rump G.C. (Hrsg.), Dialoge. Beiträge zur Interaktions- und Diskursanalyse, Hildesheim, S. 39-65.

SCHWitALLA Johannes, 1979, Dialogsteuerung in Interviews. Ansätze zu einer Theorie der Dialogsteuerung mit empirischen Untersuchungen von Politiker-, Experten- und Starinterviews in Rundfunk und Fernsehen, München.

SCHWitalla Johannes, 1987, Sprachliche Mittel der Konfliktreduzierung in Streitgesprächen, in: Schank G./Schwitalla J. (Hrsg.), Konflikte in Gesprächen, Tübingen, S. 99-175.

Schwitalla Johannes, 1996, Beziehungsdynamik. Kategorien für die Beschreibung der Beziehungsgestaltung sowie der Selbst- und Fremddarstellung in einem Streit- und Schlichtungsge- 
spräch, in: Kallmeyer W. (Hrsg.): Gesprächsrhetorik. Rhetorische Verfahren im Gesprächsprozeß, Tübingen, S. 279-349.

SPIEGEL Carmen, 2011, Streit. Eine linguistische Untersuchung verbaler Interaktion in alltäglichen Zusammenhängen, Mannheim.

\section{Quellen}

1. Der Spiegel 25/2013

Streitgespräch „Warum lobst du mich nicht“

Teilnehmer: Gesundheitsökonom Karl Lauterbach, Ärztekammerpräsident Frank Ulrich Montgomery.

2. Der Spiegel $28 / 2013$

Streitgespräch „Ach, Herr Lucke“

Teilnehmer: AfD-Chef Bernd Lucke, Linkspartei-Vizin Sahra Wagenknecht.

3. Der Spiegel 47/2013

Streitgespräch „Stich ins Herz“

Teilnehmer: Wirtschaftswissenschaftler Marcel Fratzscher, Ökonom Hans-Werner Sinn.

4. Der Spiegel 07/2014

Streitgespräch „Minderjährige im Wettbüro“

Teilnehmer: Suchttherapeut Klaus Wölfling, Spielelobbyist Martin Lorber.

5. Der Spiegel 12/2014

Streitgespräch „Das kann ich besser“

Teilnehmer: Politiker Jean-Claude Juncker und Martin Schulz.

6. Der Spiegel 31/2014

Streitgespräch „Raubtiere brauchen Fleisch“

Teilnehmer: Philosoph Jürg Luy, Zoodirektor Gunther Nogge.

7. Der Spiegel 33/2014

Streitgespräch „Wir sparen zu viel““

Teilnehmer: Wirtschaftswissenschaftler Ökonomen Thomas Mayer und Peter Bofinger.

8. Der Spiegel 47/2014

Streitgespräch „Es hat Fehler gegeben“

Teilnehmer: Autolobbyist Matthias Wissmann, Verbraucherschützer Thilo Bode.

9. Der Spiegel 49/2014

Streitgespräch „Ich war viel schlimmer als Sie“

Teilnehmer: Bundesfinanzminister Wolfgang Schäuble, CDU-Politiker Jens Spahn.

10. Der Spiegel 52/2014

Streitgespräch „Sie sind ein Scheinriese“

Teilnehmer: Sprecher des Fahrdienstes Uber Fabien Nestmann, Sprecher des Taxenverbands Clemens Grün.

11. Der Spiegel 20/2015

Streitgespräch „Es ist ein Elend“

Teilnehmer: Bayerns Innenminister Joachim Herrmann, Menschenrechtsaktivist Elias Bierdel.

12. Der Spiegel 35/2015

Streitgespräch „Ich will jetzt einen Kaiserschnitt!“

Teilnehmer: Hebamme Martina Klenk, Gynäkologe Volker Ragosch. 